\title{
TLOCI: A Fully Loaded Speckle Killing Machine
}

\author{
Christian Marois ${ }^{1}$, Carlos Correia ${ }^{1,2}$, Jean-Pierre Véran ${ }^{1}$ \\ and Thayne Currie ${ }^{3}$ \\ ${ }^{1}$ National Research Council of Canada, Dominion Astrophysical Observatory \\ 5071 West Saanich Rd, Victoria, BC, V9E 2E7, Canada \\ email: christian.marois@nrc-cnrc.gc.ca \\ ${ }^{2}$ Centre for Astrophysics of the University of Porto, Rua das Estrelas 4150-762 Porto, Portugal \\ ${ }^{3}$ Dept. of Physics and Astronomy, University of Toronto
}

\begin{abstract}
A new high-contrast imaging subtraction algorithm (TLOCI) is presented to maximize a planet signal-to-noise ratio. The technique uses an input spectrum and template PSFs to optimize the reference image coefficient determination to minimize the flux contamination via self-subtraction (thus maximizing its throughput wavelength per wavelength) of any planet that have a similar spectrum to the template spectrum in the image, while trying, at the same time, to maximize the speckle noise subtraction. The optimization is performed by a correlation matrix conditioning. Using laboratory Gemini Planet Imager data, the new algorithm is shown to be superior to the simple/double difference, polynomial fit and original LOCI algorithm.
\end{abstract}

Keywords. stars: imaging, instrumentation: adaptive optics, methods: data analysis, techniques: image processing, (stars:) planetary systems

\section{Introduction}

Direct exoplanet imaging is a challenging endeavour, as planets are located very close to their host stars and they are orders of magnitude fainter. The most successful technique to suppress the star light has been the angular differential imaging (ADI, where the intrinsic field-of-view rotation is used to decouple the light of quasi-static aberrations from real objects) in conjunction with the locally optimized combination of images (LOCI) algorithm (Marois et al. 2006, Lafrenière et al. 2007). With the development of extreme adaptive optics system integral field spectrographs, another technique that uses the intrinsic spectral differences between stars and cool planets by differencing polychromatic images (spectral differential imaging SDI, Marois et al. 2000, Sparks \& Ford 2002) can now be used. One challenge with SDI is the possible large planet flux variation as a function of wavelength. This important variation is inducing a large amount of planet self-subtraction when trying to use the original LOCI algorithm with SDI, complicating the planet detection and spectral extraction. In this proceeding, we are presenting a new algorithm (TLOCI) to perform an optimal image subtraction using any type of data acquisition scheme (such as ADI and SDI) that maximizes a planet SNR.

The new subtraction algorithm (called TLOCI for "template LOCI") differs from the original LOCI (minimizing the noise) or SDI damped-LOCI (minimizing the noise while trying to maximize the flux in an aperture of a known planet, Pueyo et al. 2012), by trying to maximize the signal-to-noise ratio (SNR) of any point sources having a specific spectrum in an image. For each LOCI section, an hypothetical planet is simulated in a noiseless image having a spectrum of interest (methane, dusty, etc). A signal correlation matrix $\left(A_{\mathrm{PSF}}\right)$ is then obtained from these simulated noiseless planet images and this matrix is added, with a proper normalizing $\Lambda$ parameter, to the standard LOCI 

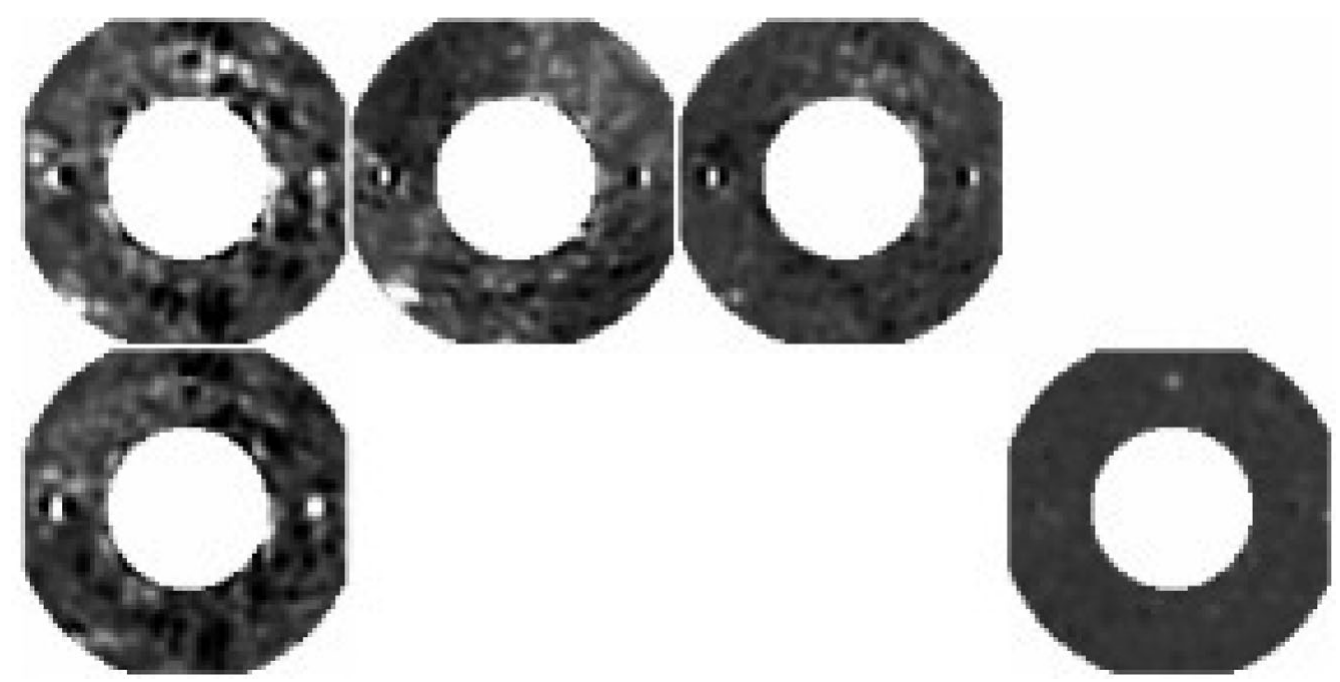

Figure 1. A $\Delta H 17.3$ methane spectrum planet discovery comparison (the planet is located North of the star; $\Delta H 14.8$ dusty (West) and flat spectrum (East) planets are also added). The first column shows a single difference (top) and double difference (bottom). A 2nd order polynomial fit with a $0.5 \lambda / D$ gap (spectral deconvolution technique) is presented in the second column. The third columns show a LOCI analysis using a $0.5 \lambda / D$ gap. The TLOCI methane-optimized algorithm is shown in the last column. TLOCI is achieving $5 \mathrm{x}$ the LOCI SNR on the methane planet (the dusty and flat spectrum planets have low SNRs in this subtraction due to the TLOCI algorithm not being optimized for these spectra).

correlation matrix $(A)$. The regularized correlation matrix $\left([1-\Lambda] A+\Lambda A_{\mathrm{PSF}}\right)$ is then inverted to obtain the LOCI coefficients that are used to compute a linear combination of images for optimized subtraction.

\section{Observations, Data Analysis \& conclusion}

The algorithm is tested using Gemini Planet Imager (GPI, Macintosh et al. 2008) laboratory data (a single $60 \mathrm{~s} \times 5$ coadd exposure, no turbulence). After initial reduction using the GPI pipeline (Maire et al. 2012), images are spatially magnified to align speckles and registered at the image center. Three planets are then implemented at 0.6 arcsec with three different spectra. One artificial planet has a flat spectrum (East), one has a methane-like spectrum (North) and the last one has a dusty-like spectrum (West). The images are then processed using various algorithms and the template-based LOCI algorithm (see Fig. 1).

The TLOCI algorithm was presented to optimally combine a set of images. This more optimal planet finding algorithm can be used for SDI, ADI or archive-based image subtraction (all these techniques performed as a single speckle subtraction step). TLOCI spectral extraction accuracy will be discussed in Marois et al., in prep.

\section{References}

Lafrenière, D., et al., 2007, ApJ, 660, 770

Macintosh, B., et al., 2008, Proceedings of the SPIE, 7015, 701518

Maire, J., et al., 2012, Proceedings of the SPIE, 7735, 773531

Marois, C., et al., 2000, PASP, 112, 91

Marois, C., et al., 2006, ApJ, 641, 556

Pueyo, L., et al., 2012, ApJS, 199, 6

Sparks, W. \& Ford, H., 2002, ApJ, 578, 543 